\title{
Synthesis and Properties of Chitosan-Silica Hybrid Aerogels
}

\author{
Michael R. Ayers, Arlon J. Hunt* \\ E.O. Lawrence Berkeley National Laboratory, Berkeley, CA, 94720, USA
}

\begin{abstract}
Chitosan, a polymer that is soluble in dilute aqueous acid, is derived from chitin, a natural polyglucosamide. Aquagels where the solid phase consists of both chitosan and silica can be easily prepared by using an acidic solution of chitosan to catalyze the hydrolysis and condensation of tetraethylorthosilicate. Gels with chitosan/TEOS mass ratios of 0.1-1.1 have been prepared by this method. Standard drying processes using $\mathrm{CO}_{2}$ give the corresponding aerogels. The amount of chitosan in the gel plays a role in the shrinkage of the aerogel during drying. Gels with the lowest chitosan/silica ratios show the most linear shrinkage, up to $24 \%$, while those with the highest ratios show only a $7 \%$ linear shrinkage. Pyrolysis at $700{ }^{\circ} \mathrm{C}$ under nitrogen produces a darkened aerogel due to the thermal decomposition of the chitosan, however, the aerogel retains its monolithic form. The pyrolyzed aerogels absorb slightly more infrared radiation in the $2-5 \mu \mathrm{m}$ region than the original aerogels. B.E.T. surface areas of these aerogels range from 470-750 $\mathrm{m}^{2} / \mathrm{g}$. Biocompatibility screening of this material shows a very high value for hemolysis, but a low value for cytotoxicity.
\end{abstract}

PACS Codes: $81.20 \mathrm{Fw} ; 81.05 \mathrm{Rm} ; 82.70 \mathrm{Gg}$

*Corresponding author. Tel: +1-510-486-5370; fax: +1-510-486-7303; e-mail: ajhunt@,lbl.gov 


\section{Introduction}

Natural materials are attractive candidates for aerogel precursors due to their potentially low cost and environmentally benign nature. Additionally, such materials may provide new application opportunities for aerogel materials. One of the most abundant organic compounds on Earth is chitin, a polyglucosamide, which is a component of the shells of crustaceans, the exoskeltons of insects, and the cell walls of fungi and certain yeasts, among other sources [1]. However, chitin in its native state is not amenable to sol gel processing due to its poor solubility. Alternatively, chitosan which is a polymer derived by the alkaline deacetylation of chitin is soluble in dilute acid.

Chitosan is a commercially available material, whose stability, chemical properties, and biocompatibility have lead to many current and potential applications. These include pharmacological, biomedical, agricultural, food, and waste treatment products [2].

Chitosan can be easily crosslinked by reagents such as glutaraldehyde, forming rigid aquagels [3]. However, all attempts to dry these gels to their corresponding aerogels were unsuccessful due to severe shrinkage and deformation of the gel. This is not surprising, as the polymer backbone of chitosan is highly polar, and capable of forming hydrogen bonds with adjacent chains, thus collapsing any low-density structure. To provide a stronger network support that would still allow a chitosan material with a high surface area to be prepared, we have investigated chitosan-silica hybrid aerogels.

\section{Experimental procedures}

The chitosan used in this work was obtained from NaturalBiopolymer, Inc. (Raymond, WA, USA.) This material (Poly $+\mathrm{L}$ grade), gives a viscosity of $\sim 20 \mathrm{cp}$ in a 1\% 
aqueous solution of acetic acid, and is $75-80 \%$ deacetylated. Tetraethoxysilane (TEOS) was reagent grade, obtained from EM Science.

Surface areas of the dried aerogels were measured with a Quantasorb (Quantachrome, Inc.) BET analyzer using 30\% $\mathrm{N}_{2} / \mathrm{He}$ for single-point BET analyses. Infrared spectra were recorded with a Perkin Elmer FTIR from $\mathrm{KBr}$ pellets. A TA Instruments SDT 3960 thermal analyses system was employed for TGA measurements. Bulk pyrolysis of aerogel monoliths was carried out in a standard tube furnace at $700{ }^{\circ} \mathrm{C}$ under an atmosphere of flowing nitrogen for $60 \mathrm{~min}$. TEM micrographs were obtained using a Topcon 00-2B microscope. Samples for microscopy were prepared by suspending a few $\mathrm{mg}$ of the aerogel in acetone, grinding the mixture, and evaporating a small amount onto holey carbon grids.

Reagent ratios used to prepare chitosan-silica gels appear in Table 1. An initial solution of chitosan in water was formed by adding a given amount of solid chitosan, the corresponding amounts of conc. $\mathrm{HCl} / \mathrm{HF}$, and ethanol, followed by vigorous stirring. This formed a viscous, pale yellow solution, to which TEOS was then added. The mixture initially contained two phases. However, after several minutes as the hydrolysis progressed, the silica containing phase was taken up by the aqueous phase. At this point the stirring was stopped and the gels poured into polystyrene molds. Gel times ranged from approximately 1-4 hours.

After standing for 48 hours, the gels were removed from the molds and soaked for 3 days in flowing deionized water. Water was removed from the gels by five 24-hour soaks in absolute ethanol. The wet gels were then converted to opaque, white aerogels using standard $\mathrm{CO}_{2}$ replacement and supercritical drying $\left(45^{\circ} \mathrm{C}\right.$ and $\left.9 \mathrm{MPa}\right)$ methods. 
Formation of hydrophobic chitosan-silica aerogels was attempted in two ways. Dried aerogels were exposed to hexamethyldisilazane (HMDS) vapors at $60{ }^{\circ} \mathrm{C}$, leading to very hydrophobic aerogels, though considerable cracking and some shrinkage occurred in all cases. Alternatively, one of the alcohol soaks prior to drying was replaced with a soak in $10 \%$ HMDS in ethanol. After supercritical drying, uncracked monoliths with very little shrinkage were obtained. However, when exposed to water these aerogels did absorb a small amount of liquid at their outer surface, though they maintained their shape and did not crack.

Biocompatibility screenings for cytotoxicity (mammal cell, direct contact) and hemolysis (rabbit blood, direct contact) were performed on hydrophobic chitosan-silica aerogels by Nelson Laboratories, Salt Lake City, UT. Analyses were performed in triplicate, using standard screening methods.

\section{Results}

Surface area and dimensional shrinkage values for aerogels with various chitosansilica ratios appear in Table 1. Soaking shrinkage refers to the linear dimensional change in the gel after water has been replaced with ethanol, while total shrinkage is similarly obtained after $\mathrm{CO}_{2}$ drying. Surface areas ranged from approximately 750 to $470 \mathrm{~m}^{2} / \mathrm{g}$, for samples with low to high chitosan contents, respectively. Total shrinkage values ranged from $\sim 23 \%$ for the samples with the lowest chitosan-silica ratio, to $\sim 7 \%$ for the sample with the highest level of chitosan. Figure 1 gives TEM images of sample 4, as prepared, and after pyrolysis at $700{ }^{\circ} \mathrm{C}$ under nitrogen. A TGA plot for the pyrolysis of sample 5 under nitrogen appears in Fig. 2. At $800^{\circ} \mathrm{C}$, the pyrolysis atmosphere was changed to air, 
to remove the remaining carbonaceous components of the aerogel. The total mass loss for this sample was $29.5 \%$. Figure 3 gives infrared spectra of sample 4 for as prepared and pyrolyzed aerogels.

Biocompatibility screening for cytotoxicity gave a score of 1 on a scale of 0 to 4 , where 0 represents no cell damage, and 4 severe cell damage. Hemolysis screening gave a result of $19.7 \%$ hemoloysis.

\section{Discussion}

The sol-gel process used to form chitosan-silica aerogels differs from standard silica aerogel preparations in that the sol is primarily aqueous. The immiscibility of TEOS with water is overcome as the monomer becomes partially hydrolyzed, and a single, clear phase is formed. In this respect, the acidic solution that is required to solubilize chitosan aids in homogenization, as acidic media favor hydrolysis of TEOS over condensation. However, to achieve reasonable gel times mixed acids are required. Acid catalyzed silica sols show extremely long gel times in all acids except HF. Using sufficient HF to dissolve the chitosan leads to a very fast reaction and difficulty in obtaining a homogenous sol. Therefore $3: 1 \mathrm{HCl} / \mathrm{HF}$ is the preferred catalyst in this system.

The physical properties of these aerogels show an interesting dependence on the ratio of chitosan to silica. As the amount of chitosan is increased, the linear shrinkage of the gel decreases significantly. This shows that chitosan inhibits gel shrinkage in spite of the fact that the polymer backbone is highly polar and capable of forming hydrogenbonded bridges between adjacent silica particles. The amount of chitosan present also 
affects the specific surface area of the dried aerogel, which decreases with increasing chitosan content. This may be due to solid impurities in the chitosan, or small portions of the polymer that did not dissolve completely in the sol-gel process. In the TEM image in Fig. 1, a sample with a fairly high chitosan content is shown. Only a microstructure typical of silica aerogels can be seen. No separate polymer phase is visible in any portion of the sample, indicating that the two phases are intimately mixed at this scale.

Chitosan, like other polysaccharides, thermally decomposes to carbon-rich materials at high temperatures under inert atmospheres. This suggests the potential for these hybrid aerogels to be used as precursors for carbon-silica composite aerogels. Pyrolysis of these aerogels at $700{ }^{\circ} \mathrm{C}$ gives black monoliths. The microstructure of the pyrolyzed aerogel is essentially identical to that of its precursor, as seen in Fig. 1. Additionally, electron diffraction of both samples showed completely amorphous behavior. The TGA plot in Fig 2 shows that approximately $18 \%$ of the mass loss occurs below $300{ }^{\circ} \mathrm{C}, 7 \%$ between 300 and $800{ }^{\circ} \mathrm{C}$, and the remaining $4.5 \%$ after oxidation by air at $800{ }^{\circ} \mathrm{C}$. Therefore, the carbon content of a pyrolyzed aerogel, originally prepared with $1.5 \mathrm{~g}$ chitosan and $5 \mathrm{~mL}$ TEOS can be estimated to be approximately $6 \%$. This total mass of the organic fraction is considerably less than the predicted value of $52 \%$ for this sample. This indicates that a moderate amount of chitosan is lost in the aerogel preparation, most likely in the aqueous soaking stages. The infrared spectra in Fig. 3 compare a pyrolyzed aerogel and its precursor. With the exception of $\mathrm{N}-\mathrm{H}$ vibrations, which are not seen in these spectra, pure chitosan shows adsorption bands at wavelengths very similar to those of silica. Therefore, the two phases are not resolved In the spectrum of the pyrolyzed aerogel, a small, but noticeable, decrease in transmission is seen 
between 2 and 6 microns, suggesting that these materials may find use as precursors for opacified aerogels.

As chitosan, is commonly used in food and drug applications, Biocompatibility screenings were performed on the aerogels to evaluate their potential use in this area. The score of 1 on the cytotoxicty test, is considered a passing score, meaning only very slight cell damage was observed during the test. However, the hemolysis results of $19.7 \%$ are very high. Any value for this test of $>5 \%$ is considered inadequate. This may result from residual fluoride ion remaining in the aerogel, or from the physical absorptive effect of the aerogel towards certain blood components. Further evaluation is required to determine this materials usefulness in biological applications.

\section{Conclusions}

Though limited to acid-catalyzed preparations, chitosan-silica composite aerogels can be easily synthesized using standard methods. The ratio of chitosan to silica leads to significant changes in the physical properties of these aerogels. These materials may find uses in diverse applications such as drug delivery, carbon silica composites via pyrolysis, and wastewater treatment using the chelating effect of the included chitosan.

\section{Acknolwedgement}

The authors wish to thank X.Y. Song for generous assistance in obtaining the TEM images reported here. 


\section{References}

[1] C. J. Brine, P. A. Sandford, J. P. Zikakis Advances in Chitin and Chitosan, Elsevier Applied Science London, New York, 1992.

[2] G. Skjak-Braek, T. Anthonsen, P. Sandford (Ed.), Chitin and Chitosan: Sources, Chemistry, Biochemistry, Physical Properties, and Applications, Elsevier Applied

Science, London, New York, 1989.

[3] W. Arguelles-Monal, F.M. Goycoolea, C. Peniche, I. Higuera-Ciapara, Polymer Gels and Networks, 6 (1998), 429 


\section{Figure Captions}

Figure 1. Top: Chitosan-silica aerogel (sample 4), Bottom: Same sample after pyrolysis at $700{ }^{\circ} \mathrm{C}$.

Figure 2. TGA plot for chitosan-silica aerogel, sample 5.

Figure 3. Infrared spectra for a) chitosan-silica aerogel (sample 4), and b) same sample after pyrolysis at $700{ }^{\circ} \mathrm{C}$. 


\section{Table 1}

Reagent ratios and physical properties for chitosan-silica aerogels.

All samples: $5 \mathrm{~mL}$ TEOS, $5 \mathrm{~mL}$ ethanol, $20 \mathrm{~mL} \mathrm{H} \mathrm{H}_{2} \mathrm{O}$

\begin{tabular}{llllllll}
\hline Sample & Chitosan $(\mathrm{g})$ & $\begin{array}{l}\text { conc. } \\
\mathrm{HCl}(\mathrm{mL})\end{array}$ & $\begin{array}{l}\text { conc. } \\
\mathrm{HF}(\mathrm{mL})\end{array}$ & $\begin{array}{l}\text { g chitosan/ } \\
\text { g silica (theory) }\end{array}$ & $\begin{array}{l}\text { Soaking } \\
\text { shrinkage } \\
(\%)\end{array}$ & $\begin{array}{l}\text { Total } \\
\text { Shrinkage } \\
(\%)\end{array}$ & $\begin{array}{l}\text { Surface } \\
\text { area } \\
\left(\mathrm{m}^{2} / \mathrm{g}\right)\end{array}$ \\
\hline 1 & 0.125 & 0.075 & 0.025 & 0.094 & 18.3 & 23.9 & 750 \\
2 & 0.250 & 0.150 & 0.050 & 0.19 & 15.5 & 18.3 & 625 \\
3 & 0.500 & 0.300 & 0.100 & 0.38 & 6.8 & 8.5 & 616 \\
4 & 1.00 & 0.600 & 0.200 & 0.75 & 5.6 & 7.0 & 608 \\
5 & 1.50 & 0.900 & 0.300 & 1.1 & 5.6 & 7.0 & 472
\end{tabular}

